\section{Shrinkage of human coronary arteries is an important determinant of de novo atherosclerotic luminal stenosis: an in vivo intravascular ultrasound study}

\author{
P C Smits, L Bos, M A Quarles van Ufford, F D Eefting, G Pasterkamp, C Borst
}

Department of

Cardiology, Heart

Lung Institute,

University Hospital,

Utrecht, Netherlands

P C Smits

L Bos

F D Eefting

G Pasterkamp

C Borst

Department of Cardiology, Thorax

Centre, Ignatius, Breda, Netherlands $M$ A Quarles van Ufford

Correspondence to: Dr Pasterkamp, Heart Lung Institute, University Hospital Utrecht, Room G02.523, Postbox 85500, 3508 GA Utrecht, Netherlands.

Accepted for publication 22 September 1997

\begin{abstract}
Objective-To assess the occurrence of arterial remodelling types and its relation with the severity of luminal stenosis in atherosclerotic coronary arteries.

Patients and methods-Twenty one de novo coronary lesions of 20 patients, who were scheduled for percutaneous transluminal coronary angioplasty (PTCA), were investigated with intravascular ultrasound before PTCA. Local arterial remodelling at the lesion site was studied by measuring the cross sectional area circumscribed by the external elastic lamina (EEL) relative to the reference site: (EEL area lesion/ reference EEL area) $\times 100 \%$. Three groups were defined. Group A: relative EEL area of less than $95 \%$ (shrinkage), group B: relative EEL area between $95 \%$ and $105 \%$ (no remodelling), group C: relative increase in EEL area of more than $105 \%$ (compensatory enlargement).

Results-All three types of remodelling were observed at the lesion site: group $A$ (shrinkage) $n=8$, group $B$ (no remodelling) $n=5$, group $C$ (compensatory enlargement) $n=8$. The mean (SD) relative EEL area at the lesion site in group $A$ and $C$ was $83(9) \%$ and $132(30) \%$, respectively. In group $A, 33 \%$ of the luminal area stenosis at the lesion site was caused by shrinkage of the artery. In contrast, group $C$ showed that $87 \%$ of the plaque area did not contribute to luminal area stenosis because of compensatory arterial enlargement.

Conclusions-These results show that both compensatory enlargement and paradoxical shrinkage occurs in the atherosclerotic coronary artery. Next to plaque accumulation, the type of atherosclerotic remodelling is an important determinant of luminal narrowing.

(Heart 1998;79:143-147)
\end{abstract}

Keywords: coronary arteries; atherosclerosis; remodelling; intravascular ultrasound

It has been shown by Glagov ${ }^{1}$ and Zarins et al that adaptive vessel wall enlargement in response to plaque accumulation occurs in the left main and left anterior descending coronary artery. Histopathological ${ }^{3}$ and intravascular ultrasound studies ${ }^{3-5}$ have shown that this process of compensatory enlargement is not limited to the left coronary artery. Pasterkamp et $a l^{3}$ have shown that in the femoral artery paradoxical arterial wall shrinkage may be observed as well. Compensatory enlargement was mainly operative in arteries with less than $25 \%$ luminal area stenosis, whereas shrinkage of the vessel wall was predominant in arteries with more than $25 \%$ luminal area stenosis. Therefore, arterial wall shrinkage may accelerate lumen narrowing in atherosclerosis. This seems to be a different mechanism of lumen obliteration than plaque formation alone and may illuminate new insights on atherosclerosis and its treatment. To determine if arterial wall shrinkage is also present in the atherosclerotic coronary artery, we performed an intravascular ultrasound study in patients before percutaneous transluminal coronary angioplasty (PTCA). Furthermore, we investigated the relation between the type of arterial wall remodelling and the severity of luminal area stenosis.

\section{Methods}

STUDY POPULATION

Twenty three patients (16 men, 7 women; mean (SD) age 59 (9)) were selected for an intravascular ultrasound study before a scheduled PTCA procedure. Only patients with single or double coronary artery disease and a significant stenosis in the proximal or mid coronary artery segment on coronary angiography were selected. Twenty four de novo atherosclerotic lesions were studied. All patients had angina pectoris class II-IV, classified according to the New York Heart Association criteria. ${ }^{6}$ Informed consent was obtained from each patient before the intravascular ultrasound procedure.

INTRAVASCULAR ULTRASOUND SYSTEM AND IMAGING PROCEDURE

The intravascular ultrasound procedures were performed with the Cardiovascular Imaging System (CVIS, Sunnyvale, California) coupled to a $2.9 \mathrm{~F}$ MicroView $30 \mathrm{MHz}$ coronary imaging catheter (CVIS, Sunnyvale, California). This mono-rail imaging catheter consists of a single rotating $30 \mathrm{MHz}$ ultrasonic transducer at the distal end of a rotating drive shaft. After coronary angiography the selected lesion was crossed with the imaging catheter over a 0.014 inch guidewire. Then the guidewire was retracted and the transducer advanced into the echolucent distal shaft $(14.5 \mathrm{~cm}$ length) of the catheter. Intravascular ultrasound images were 
Table 1 Patient characteristics

\begin{tabular}{|c|c|c|c|c|c|c|c|c|c|c|c|}
\hline Number & Sex & $\begin{array}{l}\text { Age } \\
\text { (years) }\end{array}$ & Artery & $\begin{array}{l}\text { Angina } \\
\text { class }\end{array}$ & $\begin{array}{l}\text { Post } \\
A M I\end{array}$ & $F H$ & $H C$ & $D M$ & $H T$ & NIC & Remodelling type \\
\hline 1 & $\mathrm{~F}$ & 66 & RCA & 3 & 0 & 0 & + & 0 & 0 & + & Shrinkage \\
\hline 2 & M & 72 & RCA & 3 & 0 & 0 & 0 & 0 & + & 0 & Shrinkage \\
\hline 3 & $M$ & 68 & RCA & 4 & 0 & 0 & + & 0 & 0 & 0 & Shrinkage \\
\hline 4 & $\mathrm{~F}$ & 67 & LAD & 3 & 0 & + & - & 0 & 0 & 0 & Shrinkage \\
\hline 5 & $\mathrm{~F}$ & 64 & RCA & 3 & 0 & + & - & 0 & + & + & Shrinkage \\
\hline 6 & $M$ & 50 & RCA & 2 & 0 & 0 & - & 0 & + & 0 & Shrinkage \\
\hline 7 & $M$ & 71 & RCA & 2 & + & 0 & + & 0 & 0 & + & Shrinkage \\
\hline 8 & $M$ & 52 & LAD & 4 & 0 & + & 0 & 0 & 0 & + & Shrinkage \\
\hline 9 & M & 61 & RCA & 2 & + & + & 0 & 0 & 0 & + & No remodelling \\
\hline 10 & $M$ & 48 & RCA & 3 & 0 & + & + & 0 & 0 & + & No remodelling \\
\hline 11 & $M$ & 65 & RCA & 2 & 0 & + & 0 & 0 & + & + & No remodelling \\
\hline 12 & $M$ & 38 & LAD & 4 & + & 0 & + & 0 & 0 & 0 & No remodelling \\
\hline 13 & $M$ & 51 & CXA & 3 & 0 & + & + & 0 & 0 & + & No remodelling \\
\hline 14 & M & 60 & RCA & 4 & 0 & 0 & 0 & 0 & + & + & Enlargement \\
\hline 15 & M & 69 & LAD & 3 & 0 & 0 & + & 0 & 0 & 0 & Enlargement \\
\hline 16 & $\mathrm{~F}$ & 57 & RCA & 3 & 0 & + & 0 & 0 & 0 & + & Enlargement \\
\hline 17 & $\mathrm{M}$ & 61 & LAD & 2 & + & 0 & + & 0 & + & + & Enlargement \\
\hline 18 & $\mathrm{~F}$ & 63 & RCA & 4 & + & + & + & 0 & + & 0 & Enlargement \\
\hline 19 & M & 56 & LAD & 3 & 0 & + & + & 0 & 0 & 0 & Enlargement \\
\hline 20 & $M$ & 44 & $\mathrm{RCA}^{\star}$ & 4 & + & 0 & 0 & + & 0 & + & Enlargement \\
\hline
\end{tabular}

+ , present; 0 , absent; - information not available.

*two lesions.

FH, family history of coronary artery disease; HC, hypercholesterolaemia; DM, diabetes mellitus; HT, hypertension; NIC, nicotine misuse.

obtained by slowly moving the transducer inside the echolucent shaft of the catheter back and forth across the lesion and its adjacent segments. Simultaneously with fluoroscopy, the intravascular ultrasound images and voices of the physicians were recorded on two high resolution S-VHS video tapes for offline analysis. For optimal correlation between the fluoroscopy and intravascular ultrasound images a time code was added to each signal by two synchronised video frame counters, or both video

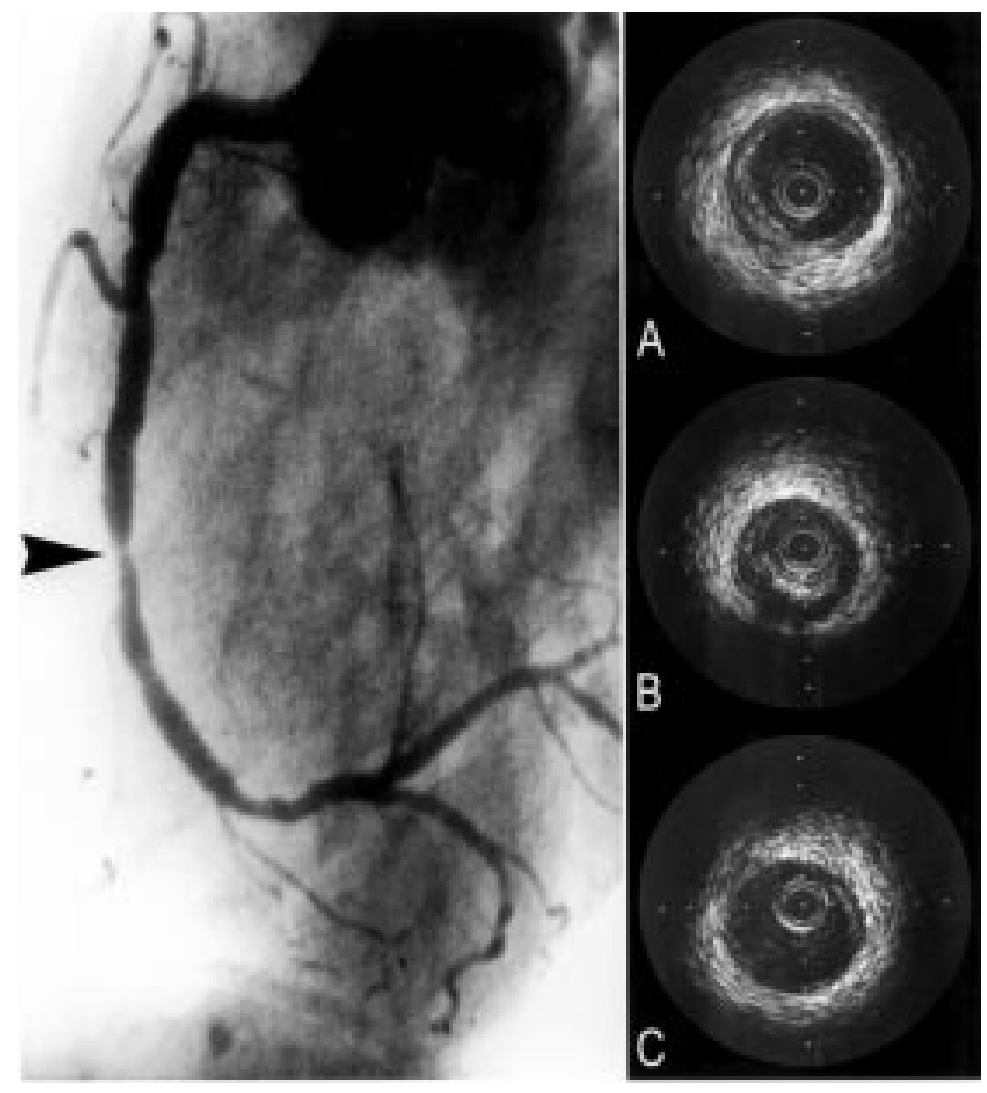

Figure 1 Angiogram and intravascular ultrasound images of the right coronary artery in one patient. The arrow indicates the lesion. The intravascular ultrasound images show the proximal reference (A), lesion (B), and distal reference $(C)$ site. At the lesion site arterial shrinkage is present. signals were online mixed into one signal and recorded.

\section{QUANTITATIVE INTRAVASCULAR ULTRASOUND} MEASUREMENTS

The intravascular ultrasound images were analysed with a digital analyser as described previously. ${ }^{7}$ In each stenosed arterial segment, three sites were selected for quantitative intravascular ultrasound analysis. The site with the most severe lumen obliteration on the intravascular ultrasound images and two reference sites in each proximal and distal adjacent segment were taken. The reference sites had no side branches in between and had no or minimal lumen narrowing on angiography and intravascular ultrasound.

In the three cross sections we traced the lumen area $\left(\mathrm{mm}^{2}\right)$ and the area circumscribed by the external elastic lamina (EEL area) $\left(\mathrm{mm}^{2}\right)$. The external elastic lamina was defined as the outer border of the echolucent zone, which represents the interface between media and adventitia. ${ }^{89}$ The media and atherosclerotic plaque area was defined as plaque area $\left(\mathrm{mm}^{2}\right)$ and calculated by subtracting the lumen area from the EEL area. Reduction of the lumen area at the lesion site could theoretically result from plaque area increase or reduction of the EEL area (arterial wall shrinkage). To compare EEL area at the lesion with its reference area we defined the reference EEL area (EEL ref) as (EEL area proximal + EEL area distal) $/ 2$. When proximal or distal side branches were present adjacent to the lesion, only the EEL area distally or proximally of the lesion was taken respectively. When large amounts of plaque were seen adjacent to the lesion only the EEL area with no or minimal plaque was used for further calculations. Arterial wall remodelling was studied by the change in EEL area (relative EEL area) and calculated as: (EEL area lesion/EEL ref area) $\times 100 \%$. The lesions were divided into three groups based on the change of EEL area. Group A: decrease in EEL area of more than 5\% 
Table 2 Baseline intravascular ultrasound (IVUS) results

\begin{tabular}{llll}
\hline & Group $A$ & Group $B$ & Group $C$ \\
\hline Lumen area $\left(\mathrm{mm}^{2}\right)$ & $15.8(5.7)(\mathrm{n}=8)$ & $13.9(1.8)(\mathrm{n}=5)$ & $13.1(2.8)(\mathrm{n}=8)$ \\
$\quad$ Proximal & $4.0(1.1)(\mathrm{n}=6)$ & $4.3(1.7)$ & $5.1(1.6)$ \\
$\quad$ Stenosis & $11.0(2.1)(\mathrm{n}=6.8)(\mathrm{n}=4)$ & $9.7(2.2)(\mathrm{n}=6)$ \\
$\quad$ Distal & $21.4(6.7)(\mathrm{n}=8)$ & $20.2(4.2)(\mathrm{n}=5)$ & $19.9(4.9)(\mathrm{n}=8)$ \\
EEL area $\left(\mathrm{mm}^{2}\right)$ & $16.5(6.0)^{\star}$ & $19.4(4.4)$ & $23.8(6.6)^{\star}$ \\
$\quad$ Proximal & $15.8(3.8)(\mathrm{n}=6)$ & $18.8(5.0)(\mathrm{n}=4)$ & $13.6(5.2)(\mathrm{n}=6)$ \\
$\quad$ Stenosis & & & \\
$\quad$ Distal & $5.6(2.2)(\mathrm{n}=8)$ & $6.4(3.3)(\mathrm{n}=5)$ & $6.8(3.2)(\mathrm{n}=8)$ \\
Plaque area $\left(\mathrm{mm}^{2}\right)$ & $13.0(6.3)$ & $15.0(3.1)$ & $19.0(6.0)$ \\
$\quad$ Proximal & $4.7(3.9)(\mathrm{n}=6)$ & $4.0(1.9)(\mathrm{n}=4)$ & $3.9(3.1)(\mathrm{n}=6)$ \\
$\quad$ Stenosis & $69.7(12.1)$ & $70.4(8.9)$ & $58.1(9.9)$ \\
$\quad$ Distal & &
\end{tabular}

Values are mean (SD).

${ }^{\star} \mathrm{p}<0.05$, group A (shrinkage) compared with group C (enlargement)

(shrinkage), group B: change in EEL area between $-5 \%$ and $5 \%$ (no remodelling), group $\mathrm{C}$ : increase in EEL area of more than 5\% (compensatory enlargement). ${ }^{10}$ Plaque contribution was defined as percentage plaque area increase to luminal area decrease at the stenosis in comparison with its reference sites and calculated by: (plaque area stenosis - plaque area reference)/(lumen area reference - lumen area stenosis $) \times 100 \%$. Per cent luminal stenosis was calculated as (1-(lumen area lesion/ lumen area reference) $) \times 100 \%$.

SELECTED ABBREVIATIONS AND DEFINITIONS EEL area $=$ area encompassed by the echodense adventitial layer. EEL area ref $=($ EEL area proximal + EEL area distal $) / 2$. Relative $\mathrm{EEL}$ area $=(\mathrm{EEL}$ area lesion $/ \mathrm{EEL}$ area ref) $\times 100 \%$. Luminal stenosis $=(1-$ (lumen area lesion/lumen area reference) $)$ $\times 100 \%$. Plaque contribution $=($ plaque area
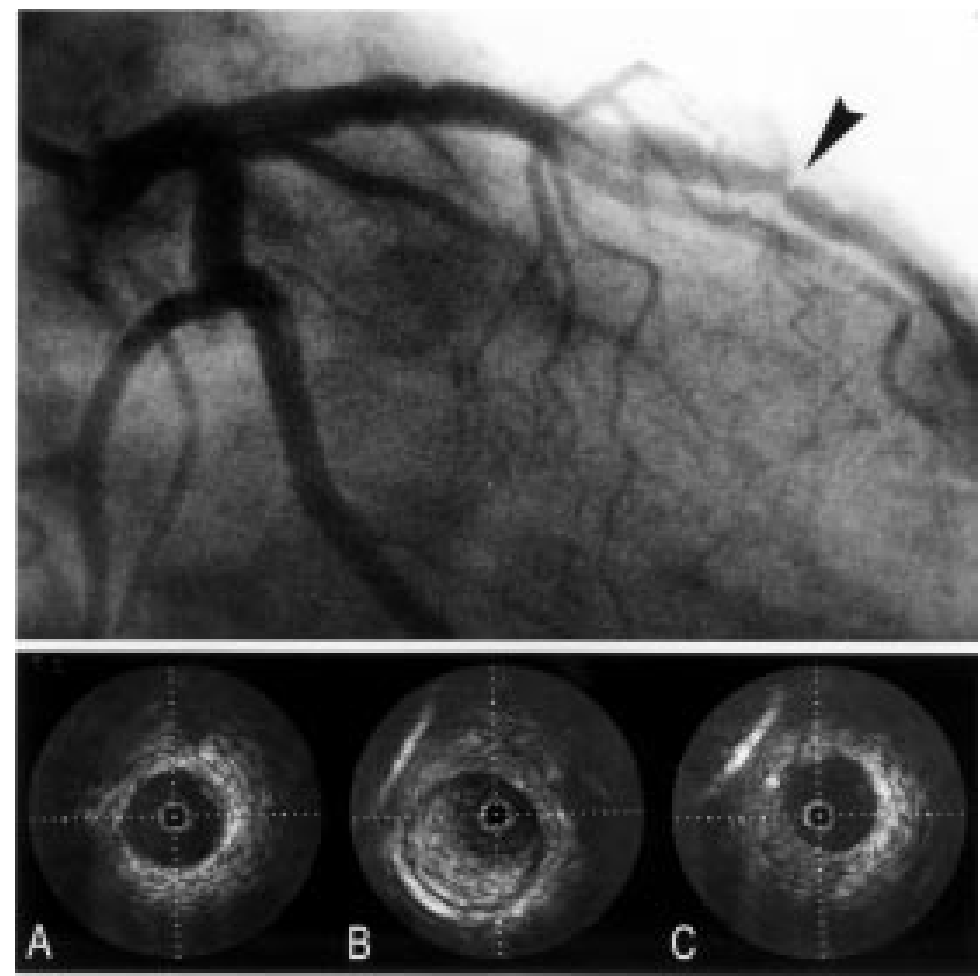

Figure 2 Angiogram and intravascular ultrasound images of the left anterior descending coronary artery in another patient. The arrow indicates the lesion. The intravascular ultrasound images show the proximal reference $(A)$, lesion $(B)$, and distal reference $(C)$ site. At the lesion site arterial compensatory enlargement is present.

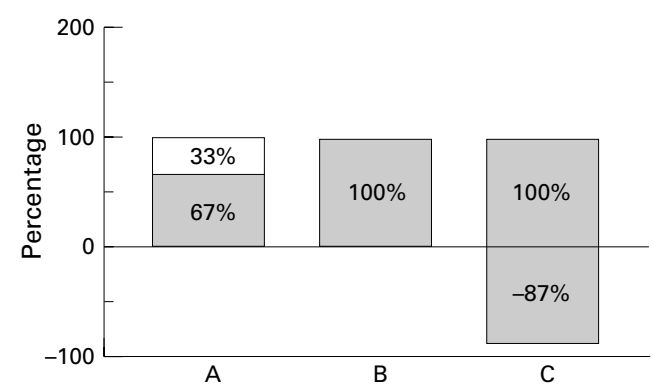

Figure 3 This bar graph represents the average percentage plaque contribution (grey bar) and the percentage remodelling contribution (white bar) to the luminal area at the lesion site by intravascular ultrasound in the different three remodelling groups. (A) shrinkage; (B)

non-remodelling; (C) compensatory enlargement group. In group $A, 67 \%$ of the luminal area decrease was contributed by plaque. The remaining $33 \%$ luminal area decrease was attributed to paradoxical shrinkage of the lesion. In group $B$ with no remodelling, the stenosis was formed alone by plaque. In group C, plaque contribution was $187 \%$ caused by compensatory enlargement of $87 \%$ in lumen area.

stenosis - plaque area reference)/(lumen area reference - lumen area stenosis) $\times 100 \%$.

STATISTICAL ANALYSIS

All values are presented as mean (SD). Comparison of data among the three different groups was performed using ordinary one way analysis of variance with Duncan range test at the post hoc test in both comparisons. A p value of less than 0.05 was considered statistically significant.

\section{Results}

Three lesions (three patients) were excluded from analysis because of insufficient intravascular image quality caused by calcification $(n=2)$, or more than $50 \%$ luminal stenosis by plaque in the reference sites $(n=1)$. Final analysis was performed on 21 lesions in 20 patients ( 15 male, five female; mean (SD) age 59 (9)). Table 1 lists the patient and lesion characteristics.

All three types of remodelling were observed at the lesion site: group A (shrinkage) $\mathrm{n}=8$, group $B$ (no remodelling) $n=5$, group $C$ (compensatory enlargement) $n=8$. Figures 1 and 2 show intravascular image examples of arterial shrinkage and compensatory enlargement, respectively. Table 2 lists the intravascular ultrasound characteristics. No statistical differences were present between the reference cross sectional areas in the different three groups. Group A showed a mean (SD) shrinkage of the vessel of $17(9) \%$, whereas group C showed a mean enlargement of $32(30) \%$

In group $A$, plaque contribution to the lumen area stenosis at the lesion site was $67 \%$ and shrinkage of the arterial wall attributed for the remaining $33 \%$. Whereas in group C, plaque contribution to the stenosis at the lesion site was $187 \%$, resulting from a $87 \%$ lumen area increase by compensatory enlargement (fig 3).

Increase of percentage luminal area stenosis with intravascular ultrasound showed a trend $(\mathrm{p}=0.051)$ towards shrinkage or lack of compensatory enlargement in the population studied (table 1). 


\section{Discussion}

In this intravascular ultrasound study, we report that coronary arteries show opposite types of arterial wall remodelling in response to atherosclerotic plaque formation. Apart from eight of $21(38 \%)$ coronary artery lesions with compensatory enlargement, we also observed eight $(38 \%)$ lesions with arterial wall shrinkage. In group A, 33\% of luminal area narrowing had to be attributed to vessel wall shrinkage.

Our results are in concordance with previous studies we performed in the femoral artery. ${ }^{3}$ An in vivo ultrasound study by Nishioka $e t$ a $l^{11}$ reported similar results with regard to different types of arterial wall remodelling (54\% compensatory enlargement, $26 \%$ shrinkage, and $20 \%$ no remodelling) in 35 primary atherosclerotic coronary lesions. They reported a similar $39 \%$ lumen stenosis by shrinkage in the group with arterial wall shrinkage.

Furthermore, we noticed a significant trend in the type of arterial wall remodelling and severity of luminal narrowing. Shrinkage dominated in lesions with severe luminal area stenosis, whereas compensatory enlargement dominated in lesions with less than 65\% luminal area stenosis. As shown by Pasterkamp et $a l^{3}$ in femoral arteries compensatory enlargement of the arterial wall dominated in lesions with less than $25 \%$ luminal area stenosis. In this study, only lesions with more than $50 \%$ luminal area stenosis were studied and compensatory enlargement was dominated in lesions with less than $65 \%$ lumen stenosis. No relation was found between the type of arterial remodelling and clinical parameters. The occurrence of unstable lesions and post infarct lesions were equally distributed among the remodelling groups.

The mechanism responsible for arterial wall remodelling is unknown. Remodelling is a focal phenomenon, which in one artery can go either way. ${ }^{3}$ Because this is not a serial intravascular ultrasound study, we did not have information about the natural history of the different lesions. Hence, it is unknown whether shrunken lesions were shrunk from the very start of the local disease or whether in time, enlarged lesions may transform into shrunken lesions and vice versa. Several mechanisms for compensatory enlargement, which seems to be a teleologically understandable response, have been proposed. ${ }^{12}$ The prevailing theory is when initial plaque growth tends to narrow the lumen, local shear stress increases and the endothelium reacts by increasing nitric oxide production with chronic vasodilatation as a consequence. ${ }^{13}$ This hypothesis seems only valid for eccentric lesions, as expansion may only be achieved by the non-diseased part of the arterial wall. Thus, adequate expansion of the atherosclerotic arterial wall may be impaired or impossible in concentric lesions. The mechanism of paradoxical shrinkage is even less understood. Perhaps the presence of circumferential dysfunctional endothelium or basal release of a local vasoconstrictor agent may attribute to arterial wall shrinkage. ${ }^{14}{ }^{15}$

Arterial wall remodelling and in particular shrinkage sheds new light on the process of luminal narrowing by atherosclerosis and may have therapeutic consequences. Hence, it is conceivable that the initial stenosis that is influenced by different remodelling mechanisms, may respond differently to angioplasty and may be more or less prone to restenosis. In restenosis, shrinkage may be the most important mechanism instead of intima hyperplasia. $^{1617}$

\section{STUDY LIMITATIONS}

The comparatively large number of right coronary artery lesions studied (14 of 21) may have influenced the presence and rate of arterial wall remodelling. In a histopathological study of human coronary arteries some differences in grade of arterial wall remodelling among the arteries occur. ${ }^{18}$ Furthermore, the reference site contained a certain amount of plaque (table 2). This may have provoked some arterial wall remodelling at the reference site that could have influenced the calculated degree of relative change in EEL area at the lesion site.

Crossing of the stenotic lesion with the ultrasound catheter could have caused a further drop in post stenotic pressure and therefore could have influenced the distal reference cross sectional area measurements. Furthermore, when side branches were present proximal or distal from the lesion only one reference cross section was taken. Subsequently tapering of the vessel segment was not taken into account. However, when we corrected for the degree of tapering as observed by Javier et $a l^{19}$ - that is, a $10 \%$ loss of vessel size (EEL area) over $10 \mathrm{~mm}$ of arterial length from the proximal reference point - then still eight of the $21(38 \%)$ analysed lesions would be categorised as being "shrunken".

In conclusion, paradoxical arterial shrinkage occurs in the human coronary artery and seems to be an important determinant of severe lumen narrowing.

1 Glagov S, Weisenberg E, Zarins CK, et al. Compensatory nlargement of human atherosclerotic coronary arteries. $N$ Engl f Med 1987;316:1371-5.

2 Zarins CK, Weisenberg E, Kolettis G, et al. Differential enlargement of artery segments in response to enlarging atherosclerotic plaques. F Vasc Surg 1988;7:386-94

3 Pasterkamp G, Wensing PJW, Post MJ, et al. Paradoxical arterial wall shrinkage may contribute to luminal narrowing of human atherosclerotic femoral arteries. Circulation 1995;91:1444-9.

4 Hermiller JB, Tenaglia AN, Kisslo $\mathrm{KB}$, et al. In vivo validation of compensatory enlargement of atherosclerotic coronary arteries. Am f Cardiol 1993;71:665-8.

5 Losordo DW, Rosenfield K, Kaufman J, et al. Focal compensatory enlargement of human arteries in response to progressive atherosclerosis. Circulation 1994;89:2570-7.

6 The Criteria Committee of the New York Heart Association. Diseases of the heart and blood vessels; nomenclature and criteria for diagnosis. 6th ed. Boston: Little, Brown, 1964.

7 Wenguang L, Gussenhoven WJ, Zhong Y, et al. Validation of quantitative analysis of intravascular ultrasound images. Int f Card Imaging 1991;6:247-53.

8 Gussenhoven EJ, Essed CE, Lancee CT, et al. Arterial wall characteristics determined by intravascular ultrasound imaging: an in vitro study. $\mathcal{F}$ Am Coll Cardiol 1989;14:94752.

9 Potkin BN, Bartorelli AL, Gessert JM, et al. Coronary artery imaging with intravascular high-frequency ultrasound. Circulation 1990;81:1575-85.

10 Pasterkamp G, Borst C, Gussenhoven EJ, et al. Remodeling of de novo atherosclerotic lesions in femoral arteries: impact on the mechanism of balloon angioplasty. $7 \mathrm{Am}$ Coll Cardiol 1995;26:422-8.

11 Nishioka T, Luo H, Eigler NL, et al. Contribution of inadequate compensatory enlargement to development of human coronary artery stenosis: an in vivo intravascular ultrasound study. 7 Am Coll Cardiol 1996;27:1571-6.

12 Post MJ, Borst C, Pasterkamp G, et al. Arterial remodeling in atherosclerosis and restenosis: a vague concept of a distinct phenomenon. Atherosclerosis 1995;118:S115-23. 
13 Lamontagne D, Pohl U, Busse R. Mechanical deformation of vessel wall and shear stress determine the basal release of endothelium-derived relaxing factor in the intact rabbit coronary vascular bed. Circ Res 1992;70:123-30.

14 Langille BL, O'Donnell F. Reduction in arterial diameter produced by chronic decreases in blood flow are endothelium-dependant. Science 1986;231:405-7.

15 Lerman A, Holmes D, Bell M, et al. Endothelin in coronary endothelial dysfunction and early atherosclerosis in humans. Circulation 1995;92:2426-31.

16 Post MJ, Borst C, Kuntz RE. The relative importance of arterial remodeling compared with intimal hyperplasia in lumen narrowing after balloon angioplasty. Circulation 1994;89:2816-21

17 Mintz GS, Popma JJ, Pichard AD, et al. Arterial remodeling after coronary angioplasty: a serial intravascular ultrasound study. Circulation 1996;94:35-43.

18 Eefting FD, Pasterkamp G, Clarijs JAGM, et al. Atherosclerotic arterial wall remodeling: a determinant of luminal narrowing in human coronary artery. Coron Artery Dis 1997;8:415-21.

19 Javier SP, Mintz GS, Popma JJ, et al. Intravascular ultrasound assessment of the magnitude and mechanism of coronary artery and lumen tapering. Am f Cardiol 1995; 75: 